\title{
Changes of dissolved organic matter derived from rape straw by Selenium pretreatment improve the inhibition of Sclerotinia sclerotiorum growth (Abstract)
}

\author{
Xiaohu Zhao \\ College of Resources and Environment, Huazhong Agricultural University, Wuhan, China \\ Corresponding author: xhzhao@mail.hzau.edu.cn
}

\begin{abstract}
In this study, dissolved organic matter (DOM) was extracted from rape straw pretreated with Se in soil to examine the inhibitory effect on the growth of Sclerotinia sclerotiorum by poisoned culture medium technique. The results revealed that DOM in culture medium inhibited the growth of $S$. sclerotiorum significantly, while the DOM derived from rape straw pretreated with Se achieved the better inhibitory effect. To confirm that Se pretreatment in soil caused changes in DOM derived from rape straw which increased antifungal effect on $S$. sclerotiorum, a two-year laboratory pot culture experiment with four levels of Se (control,

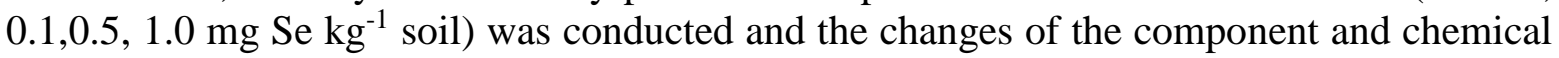
structure in DOM were investigated using UV-visible spectroscopy, fluorescent spectroscopy and FTIR spectroscopy. Results of spectrograms showed that application of Se in soil altered the DOM properties, such as getting more irreplacable aromatic structure in substituent groups of DOM, achieving less conjugated systems, and gaining more alcohol, phenol, amine, amino acid salt and alkyne in DOM, and all the changes of DOM chemical composition improved the inhibition of $S$. sclerotiorum growth. The results of this study not only provide agricultural environmental safety information for more effective utilization of rape straw, but also light another approach of Se application in agricultural science.
\end{abstract}

Keywords: Selenium, rape straw, dissolved organic matter (DOM), composition, inhibition, Sclerotinia sclerotiorum. 
